\title{
Low-dose CT with tin filter combined with iterative metal artefact reduction for guiding lung biopsy
}

\author{
Jing Zhang ${ }^{\#}$, Meiling Liü^${ }^{\sharp}$, Daihong Liu^, Xiaoqin Li^, Meng Lin^, Yong Tan^, Yuesheng Luo^, \\ Xiangfei Zeng^, Hong Yu^, Hesong Shen^, Xiaoxia Wang^, Leilei Liu^, Yuchuan Tan^, Jiuquan Zhang^ \\ Department of Radiology, Chongqing University Cancer Hospital, Chongqing, China
}

Contributions: (I) Conception and design: J Zhang, J Zhang; (II) Administrative support: J Zhang, M Lin, Y Tan; (III) Provision of study materials or patients: M Liu, D Liu, X Li, Y Luo; (IV) Collection and assembly of data: M Liu, Y Luo, X Zeng, H Yu, H Shen, X Wang, L Liu, Y Tan; (V) Data analysis and interpretation: J Zhang, M Liu, D Liu, X Li; (VI) Manuscript writing: All authors; (VII) Final approval of manuscript: All authors.

"These authors contributed equally to this work.

Correspondence to: Prof. Jiuquan Zhang. Department of Radiology, Chongqing University Cancer Hospital \& Chongqing Cancer Institute \& Chongqing Cancer Hospital, 181 Hanyu Road, Shapingba District, Chongqing 400030, China. Email: zhangjq_radiol@foxmail.com.

Background: Computed tomography (CT) is currently the imaging modality of choice for guiding pulmonary percutaneous procedures. The use of a tin filter allows low-energy photons to be absorbed which contribute little to image quality but increases the radiation dose that a patient receives. Iterative metal artefact reduction (iMAR) was developed to diminish metal artefacts. This study investigated the impact of using tin filtration combined with an iMAR algorithm on dose reduction and image quality in CT-guided lung biopsy.

Methods: Ninety-nine consecutive patients undergoing CT-guided lung biopsy were randomly assigned to routine-dose CT protocols (groups A and B; without and with iMAR, respectively) or tin filter CT protocols (groups C and D; without or with iMAR, respectively). Subjective image quality was analysed using a 5-point Likert scale. Objective image quality was assessed, and the noise, contrast-to-noise ratio, and figure of merit were compared among the four groups. Metal artefacts were quantified using CT number reduction and metal diameter blurring. The radiation doses, diagnostic performance, and complication rates were also estimated.

Results: The subjective image quality of the two scan types was compared. Images with iMAR reconstruction were superior to those without iMAR reconstruction (group A: $3.49 \pm 0.65$ vs. group B: $4.63 \pm 0.57 ; \mathrm{P}<0.001$, and group $\mathrm{C}: 3.88 \pm 0.66$ vs. group $\mathrm{D}: 4.82 \pm 0.39 ; \mathrm{P}<0.001)$. Images taken with a tin filter were found to have a significantly higher figure-of-merit than those taken without a tin filter (group A: $14,041 \pm 7,230$ vs. group C: $21,866 \pm 10,656 ; \mathrm{P}=0.001$, and group B: $13,836 \pm 6,849$ vs. group D: 21,639 $\pm 9,964$; $\mathrm{P}=0.001)$. In terms of metal artefact reduction, tin filtration combined with iMAR showed the lowest CT number reduction $(116.62 \pm 103.48 \mathrm{HU})$ and metal diameter blurring $(0.85 \pm 0.30)$ among the protocols. The effective radiation dose in the tin filter groups was $73.2 \%$ lower than that in the routine-dose groups. The complication rate and diagnostic performance (sensitivity, specificity, and overall accuracy) did not differ significantly between the tin filter and routine-dose groups (all $\mathrm{P}>0.05$ ).

$\wedge$ ORCID: Jing Zhang, 0000-0003-3883-7132; Meiling Liu, 0000-0003-4446-3974; Daihong Liu, 0000-0001-5606-4394; Xiaoqin Li, 00000001-9238-7924; Meng Lin, 0000-0002-1245-5665; Yong Tan, 0000-0002-5824-6754; Yuesheng Luo, 0000-0002-5954-7305; Xiangfei Zeng, 0000-0001-7192-6079; Hong Yu, 0000-0001-5961-4275; Hesong Shen, 0000-0003-4467-0404; Xiaoxia Wang, 0000-0002-95400677; Leilei Liu, 0000-0002-7134-9491; Yuchuan Tan, 0000-0001-7097-7448; Jiuquan Zhang, 0000-0003-0239-6988. 


\begin{abstract}
Conclusions: Tin filtration combined with an iMAR algorithm may reduce the radiation dose compared to the routine-dose CT protocol, while maintaining comparable diagnostic accuracy and image quality and producing fewer metal artefacts.
\end{abstract}

Keywords: Lung; biopsy; radiation dosage; metal artefacts; tin filtration

Submitted May 28, 2021. Accepted for publication Oct 08, 2021.

doi: 10.21037/qims-21-555

View this article at: https://dx.doi.org/10.21037/qims-21-555

\section{Introduction}

Lung cancer has been responsible for more deaths in the $21^{\text {st }}$ century than any other malignancy and has a high incidence rate (1). Collecting histological samples is crucial for pulmonary lesion diagnosis and therapy planning. Owing to its high spatial and inherent contrast resolutions of the lung parenchyma, computed tomography (CT) is currently the imaging modality of choice for guiding pulmonary percutaneous procedures (2). However, an increase in the cumulative effective radiation dose may significantly increase the risk of cancer, and metal artefacts can dramatically obscure critical structures.

Unlike a standard CT protocol, CT-guided biopsy requires repeated CT scans to localise the lesion, adjust the needle tip, and review any biopsy-associated complications, resulting in a significantly higher radiation dose than a standard CT protocol. Based on the "as low as reasonably achievable" (ALARA) principle, patients should receive the lowest possible radiation dose in clinical procedures (3). Various methods to minimise the exposure dose have been adopted, including lowering the tube potential and/or current, using automated exposure control, using selective in-plane shielding, and using iterative image reconstruction (4-8). However, lowering the tube potential may reduce ray penetration and cause more metal artefacts, resulting in a reduced signal-to-noise rate and lower image quality.

The development of CT hardware and software has led to new clinical-use CT equipment with built-in tin filters for spectral shaping. When placed in front of the $\mathrm{X}$-ray tube, the tin filter absorbs low-energy photons that contribute little to image quality and increase the radiation dose received by the patient (9). Previous studies on third-generation dual-source CT with tin filtration have evidenced that non-contrast chest CT can achieve high image quality and a good view of anatomical structures while decreasing the radiation dose by exploiting the naturally high contrast of the lung $(10,11)$.
Metal artefacts include photon starvation artefacts and beam hardening artefacts. Photon starvation artefacts are caused by strong absorption and insufficient photon transmission, whereas beam hardening artefacts result from the additional absorption of low-energy photons. Using a tin filter to reduce metal artefacts excludes the low-energy photons that are primarily responsible for beam hardening and photon starvation. This can improve the effective energy and lead to a substantial decrease in artefact severity (12). Recently, a new dedicated metal artefact reduction postprocessing algorithm-iterative metal artefact reduction (iMAR) - was developed to further reduce metal artefacts, thus improving the visualisation of the biopsy target. iMAR is an efficient technique that can be used in many situations, including for patients with hip prostheses (13), spinal instrumentation (14), and CT-guided microwave ablation (15).

Theoretically, radiation doses and metal artefacts can be reduced using tin filtration combined with an iMAR algorithm. iMAR can be adapted and applied to the data acquired using tin filtration in the biopsy procedure. We hypothesised that tin filtration combined with an iMAR algorithm may reduce the radiation dose and increase the image quality while maintaining good overall diagnostic accuracy. Therefore, this study aimed to investigate the impact of using tin filtration combined with an iMAR algorithm on radiation dose reduction, image quality, and biopsy outcomes in patients undergoing CT-guided biopsy procedures.

The following article is presented in accordance with the STARD reporting checklist (available at https://dx.doi. org/10.21037/qims-21-555).

\section{Methods}

\section{Study population}

Between April 2019 and July 2020, all consecutive patients 


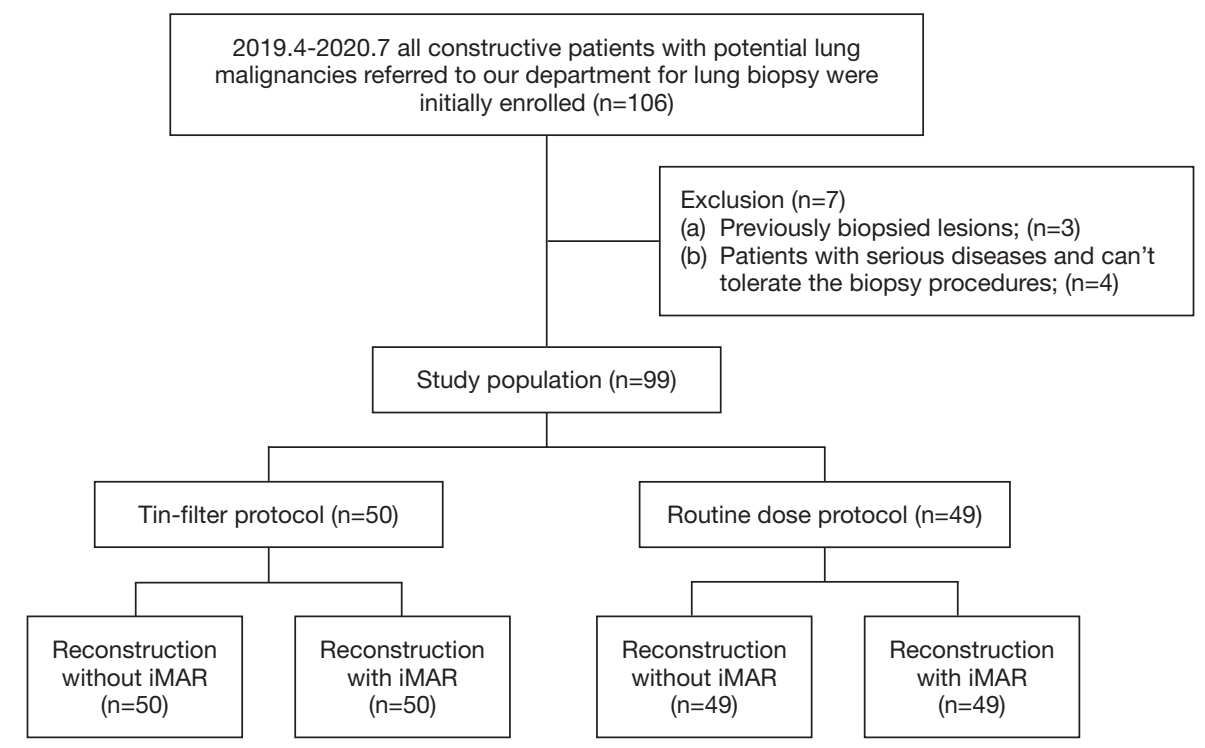

Figure 1 Flowchart for the enrolment of study participants. SAFIRE, sinogram-affirmed iterative reconstruction; iMAR, iterative metal artefact reduction.

Table 1 Acquisition parameters

\begin{tabular}{lcc}
\hline Parameters & $\begin{array}{c}\text { Routine-dose } \\
\text { protocol }\end{array}$ & $\begin{array}{c}\text { Tin filter } \\
\text { protocol }\end{array}$ \\
\hline kV & 130 & Sn130 \\
Ref mAs & $56 \mathrm{mAs}$ & $56 \mathrm{mAs}$ \\
Rotation time (s) & 0.5 & 0.5 \\
Increment collimation $(\mathrm{mm})$ & $16 \times 0.75$ & $16 \times 0.75$ \\
Pitch & 1.5 & 1.5 \\
Section thickness $(\mathrm{mm})$ & 1.5 & 1.5 \\
Increment $(\mathrm{mm})$ & 1.2 & 1.2 \\
Field of view $\left(\mathrm{mm}^{2}\right)$ & $400 \times 400$ & $400 \times 400$ \\
Matrix & $512 \times 512$ & $512 \times 512$ \\
\hline
\end{tabular}

with potential lung malignancies who were referred to Radiology department in Chongqing University Cancer Hospital for lung biopsy were prospectively selected for the study. Initially, 106 patients were found to be eligible. The study exclusion criteria were as follows: (I) patients with previously biopsied lesions $(\mathrm{n}=3)$, and (II) patients with serious diseases of the heart, lungs, or kidneys, or coagulation disorders $(n=4)$. After the initial screening, 99 patients were enrolled in our study and were randomly assigned to either a tin filter protocol or a routine-dose protocol (Figure 1). No biopsy had to be repeated in any of the patients.

The study was conducted in accordance with the Declaration of Helsinki (as revised in 2013). This study was approved by the ethics committee of Chongqing University Cancer Hospital (approval No. CZLS2021182-A), and all the participants provided written informed consent after receiving a detailed explanation of the procedure.

\section{CT acquisition}

A common clinical-use CT scanner (SOMATOM go.Up, Siemens Healthcare, Erlangen, Germany) was employed in this study. The scan parameters for the tin filter protocol were as follows: tube voltage $=S n 130 \mathrm{kV}$, tube current $=56 \mathrm{~mA} / \mathrm{s}$, increment collimation $=16 \times 0.75 \mathrm{~mm}$, pitch $=1.5$, rotation time $=0.5 \mathrm{~s}$. The scan parameters for the routine-dose protocol were as follows: tube voltage $=130 \mathrm{kV}$, tube current $=56 \mathrm{~mA} / \mathrm{s}$, increment collimation $=16 \times 0.75 \mathrm{~mm}$, pitch $=1.5$, rotation time $=0.5 \mathrm{~s}$ (Table 1$)$.

\section{Image reconstruction}

The images were reconstructed using sinogram-affirmed iterative reconstruction (SAFIRE) with and without iMAR for each scan during the biopsy procedure. Additionally, all the images were reconstructed using SAFIRE at a strength 

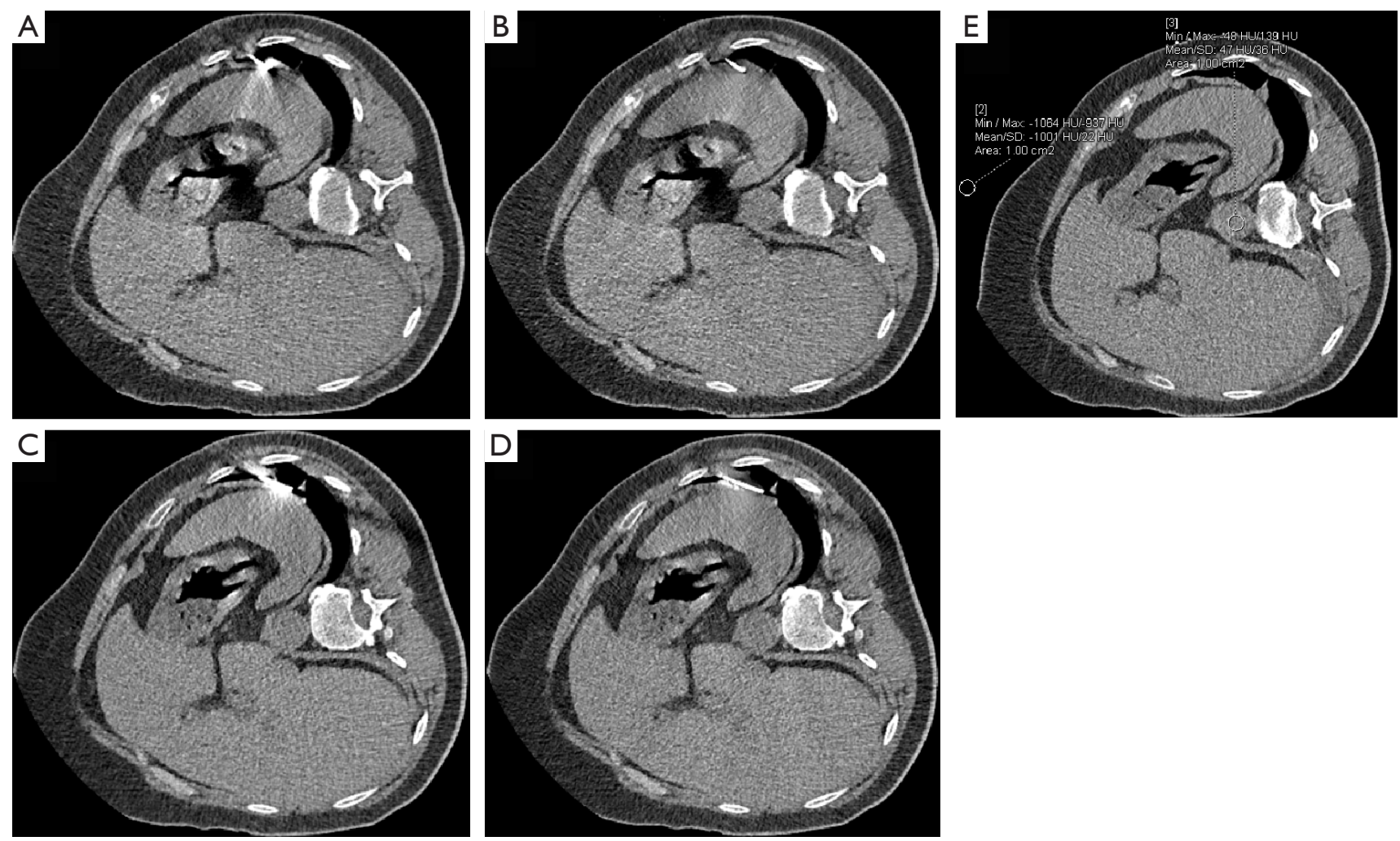

Figure 2 CT-guided biopsy of small pulmonary nodules near the diaphragm. Images were reconstructed without (A,C) and with (B,D) iMAR. The needle tip entered the diaphragm in the wrong direction initially (A,B), and then the needle was inserted into the target lesion after several adjustments (C,D). The needle tip is visualised more precisely on images with iMAR (B,D) than that without iMAR (A,C). The mean CT attenuation values (HU) and image noise (standard deviation (SD) of measured HU values) were determined using equal $\left(1.0 \mathrm{~cm}^{2}\right)$ similar ROIs for all patients, placed within the thoracic aorta and air space outside of the anterior chest wall (E). iMAR, iterative metal artefact reduction.

level of 3, with a slice thickness of $1.5 \mathrm{~mm}$ and an increment of $1.2 \mathrm{~mm}$. Reconstructions using a dedicated soft-tissue convolution kernel (Br40) and a lung convolution kernel (B60) were generated. The reconstructed field of view was $400 \mathrm{~mm} \times 400 \mathrm{~mm}$, and the image matrix was $512 \times 512$ pixels. The reconstruction orientation was axial with an identical field of view (Table 1).

Four image types were generated. Images obtained using the routine-dose protocol and reconstructed without and with iMAR were assigned to groups $\mathrm{A}$ and $\mathrm{B}$, respectively. Images obtained using the tin filter protocol and reconstructed without and with iMAR were assigned to groups $\mathrm{C}$ and $\mathrm{D}$, respectively.

\section{CT-guided biopsy procedures}

Depending on the location of the lesions, the patient was placed in the supine, prone, or lateral decubitus position. Needle pathways were selected based on a pre-procedural CT scan to avoid any necrotic regions. The scan area was limited to the lesion area, and the free-breathing mode was employed to decrease the psychological burden on patients. After each needle adjustment step, the needle position was determined by scanning the lesion area using the pre-set CT scan protocols (Figure 2). Once the tip of the needle entered the lesion, samples were obtained. To assess post-procedural complications, after the removal of the needle, one final post-biopsy scan of the whole lung was obtained from each patient using routine-dose parameters. The sample was then sent to the pathology department immediately.

All the biopsies were performed using an 18-gauge automatic cutting needle, with an inner stylet surrounded by a 17-gauge outer needle (Bard Magnum, Bard Medical, NJ, USA). During the biopsy procedure, the outer needle 

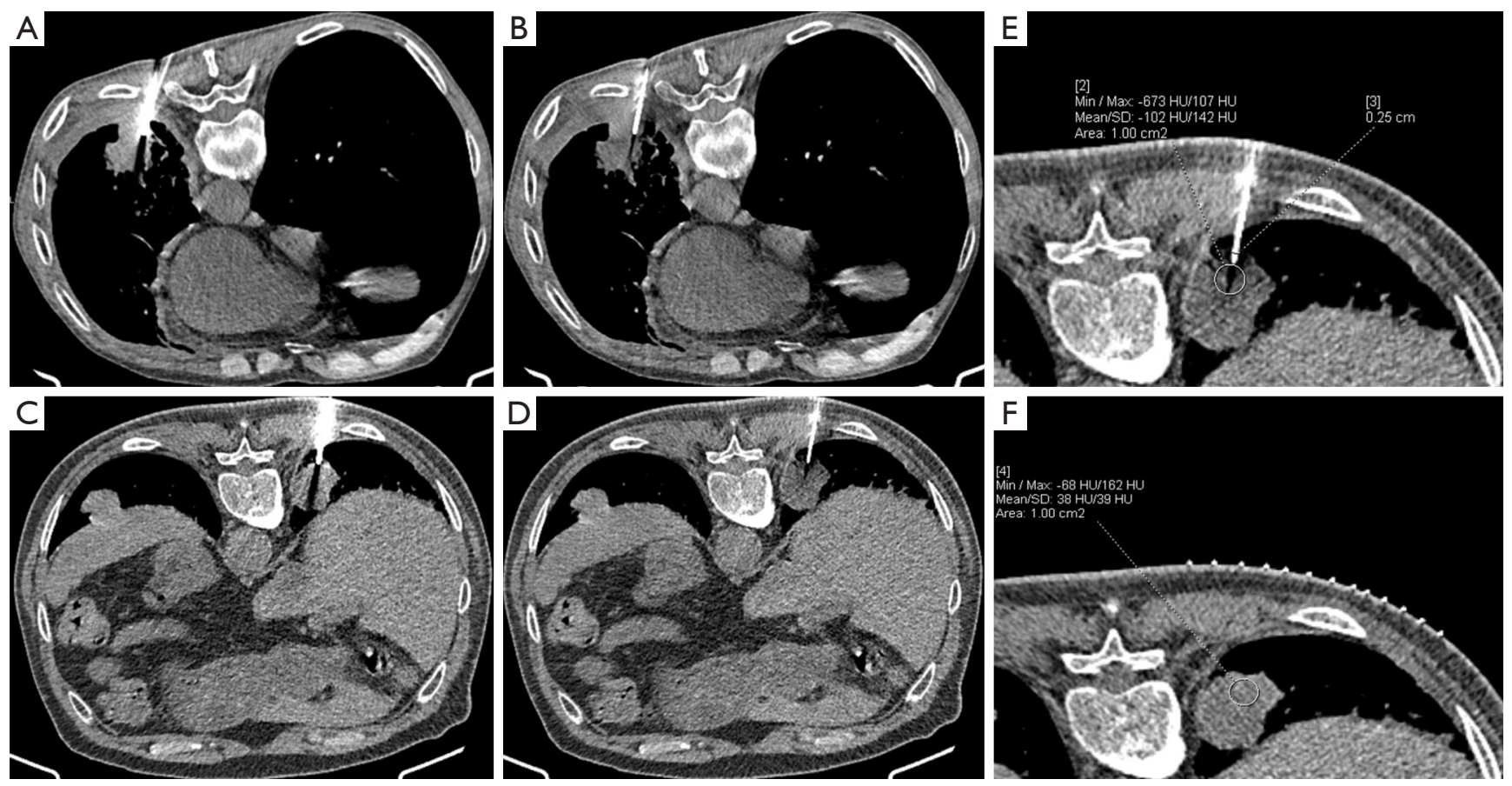

Figure 3 Four types of images acquired from two patients (A-D), and the illustration of how CT number reduction (CTNR) and metal diameter blurring (MDB) were measured (E,F). (A,B) Axial images of routine-dose CT (tube voltage = $130 \mathrm{kV}$; CTDIvol =6.01 mGy; DLP $=74 \mathrm{mGy} \cdot \mathrm{cm} ; \mathrm{ED}=1.04 \mathrm{mSv}$ ) of a 72-year-old man with a pulmonary nodule in the right lower lobe. (C,D) Axial images of tin filter CT (tube voltage $=\mathrm{Sn} 130 \mathrm{kV}$; CTDIvol $=2.86 \mathrm{mGy}$; DLP $=35 \mathrm{mGy} \cdot \mathrm{cm}$; ED $=0.49 \mathrm{mSv}$ ) of a 62 -year-old man with a pulmonary nodule in the left lower lobe. (A) Routine-dose CT scan without iMAR with a subjective image score of 3; (B) routine-dose CT scan with iMAR with a subjective image score of 4; (C) low-dose CT scan without iMAR with a subjective image rate of 4; (D) low-dose CT scan with iMAR, with a subjective image score of 5 . The image quality with iMAR was superior to that without iMAR. The image quality was comparable between both protocols despite a substantial dose reduction using spectral shaping. The mean CT attenuation values over the ROI was measured using the pre-procedural CT scan (F) and scan that included metal (E). The width of the needle profile was measured at the cross-section (E). CTDIvol, CT dose index; DLP, dose length product; ED, effective dose; iMAR, iterative metal artefact reduction.

was used for lesion localisation and the stylet for sample collection; the outer needle was advanced using a trigger.

\section{Subjective image quality evaluation}

As differences in work arrangements and operators may lead to variation in the assessment of subjective image quality, the image quality evaluation in this study was carried out by two chest radiologists (with 3 and 5 years of experience, respectively), rather than by the intervention operators. The two radiologists were blinded to the study participants' clinical information and performed the image quality evaluation independently. The overall image quality, including the visual region of interest (ROI) in the target lesion and the sharpness of the vessels in the proposed needle pathway, was assessed. The overall image quality was graded using a 5 -point scoring system: $1=$ unacceptable (poor definition of lesion and access), 2 = poor (borderline adequate lesion visualisation but inadequate visualisation of safe access, needle tip, or target area), 3 = adequate (adequate definition of lesion, access, and needle tip; slight impact of image noise), 4 = good (good visualisation of lesion, access, and needle tip; minimal image noise), and $5=$ excellent (excellent definition of lesion, access, and needle tip). Scans with a quality score of $3-5$ were considered to be acceptable (Figure $3 A-3 D$ ).

\section{Objective image quality evaluation}

All values were measured on the soft-tissue convolution kernel (Br40) image. CT values (HU) and image noise [standard deviation (SD) of measured $\mathrm{HU}$ values] were determined on the reconstructed $1.5-\mathrm{mm}$ axial CT images 
using similar $\left(1.0 \mathrm{~cm}^{2}\right)$ ROIs within the thoracic aorta and the air space outside the anterior chest wall for all patients (Figure 2E). Each ROI was placed in an identical or almost identical segment by two operators (with 5 and 3 years of experience in CT imaging, respectively). Contrast in chest CT mainly exists due to the difference in attenuation between the pulmonary vasculature, the surrounding parenchyma, and air. As lung tissue could not be obtained from the same area in different patients due to the limitation of scanning range and individual heterogeneity, image noise was considered as the SD of CT attenuation and was measured within the air space outside the anterior chest wall. Finally, the contrast-to-noise ratio (CNR) and figureof-merit (FOM) were calculated for each image dataset using the equations below. The FOM is a metric used to compare the dose efficiency of two scanning protocols.

$$
\begin{aligned}
& \mathrm{CNR}=\frac{\text { mean } \mathrm{CT} \text { value }(\text { thoracic aorta })-\text { mean } \mathrm{CT} \text { value }(\text { air })}{\mathrm{SD}(\text { air })} \\
& \mathrm{FOM}=\frac{\mathrm{CNR} 2}{\text { Effective Dose }}
\end{aligned}
$$

\section{Metal artefact evaluation}

Metal artefacts were quantified using CT number reduction (CTNR) and metal diameter blurring (MDB) at the soft tissue window. The mean CT number of lesions was obtained using a 10 -mm-diameter ROI at the lesions with the most obvious streak artefacts. The mean CT number over the ROI was measured using the pre-procedural CT scan (no metal) and a second scan that included metal (Figure $3 E, 3 F)$. The CTNR was then calculated for each metal scan using the following equation: CTNR $=\mathrm{HU}$ (metal-image) HU (baseline image). The outer needle was evaluated. The metal diameter accuracy was calculated from the image data by measuring the width of the needle profile at the crosssections (Figure $3 E$ ) and comparing it to the true outer diameter of the actual needle $(16,17)$ using the following equation: $\mathrm{MDB}=($ Width (image) - Width (true) $/$ Width (true)). Lower values for CTNR and MDB indicate fewer artefacts and higher image quality.

\section{Radiation dose}

The volume CT dose index (CTDIvol) and dose length product (DLP) for each scan were obtained from the patient dose reports. The effective dose (ED) was calculated by multiplying the DLP by the conversion factor $(0.014 \mathrm{mSv} / \mathrm{mGy} \cdot \mathrm{cm})$.

\section{Diagnostic performance and complication rates}

Clinical data, including the pathological biopsy diagnosis, were obtained from the hospital information system. Biopsy diagnoses were assigned to one of four categories: malignancies, suspected malignancies, specific benign lesions, or non-specific benign lesions. The final diagnoses for suspected malignancy or non-specific benign lesions were established based on surgical or follow-up results, and information related to the biopsy procedure was recorded by technicians. The parameters are listed in Table 2 .

\section{Statistical analysis}

Statistical analysis was performed using SPSS 22.0 (IBM Corp., Armonk, NY, USA), with the significance level set at 0.05 . Data were displayed as the mean $\pm \mathrm{SD}$ and tested for normal distribution using the Shapiro-Wilk test. The noise was normally distributed, so Student's $t$-test and analysis of variance were employed. The CNR, FOM, CTNR, MDB, CTDIvol, and DLP data were not normally distributed, so the Mann-Whitney U test and Kruskal-Wallis test were employed. Categorical data were computed using Pearson's chi-square test and Fisher's exact test.

Interobserver agreement for subjective image quality and retest reliability for quantitative analysis were assessed using the weighted kappa $(\kappa)$ and interclass correlation coefficient (ICC).

\section{Results}

\section{Baseline characteristics}

The characteristics of the patients and procedures are summarised in Table 2. No significant differences were found between the tin filter and routine-dose groups in terms of mean age $(60.62 \pm 9.68 v s$. $63.92 \pm 8.84, \mathrm{P}=0.080)$, sex distribution $(\mathrm{P}=0.592)$, or body mass index $(\mathrm{BMI})$ $(22.98 \pm 4.40$ vs. $23.46 \pm 3.06, \mathrm{P}=0.532)$. The mean lesion size was 4.44 and $3.76 \mathrm{~cm}$ in the tin filter and routine-dose groups, respectively $(\mathrm{P}=0.179)$. The lesion location, pleuralto-lesion depth, and patient position during biopsy did not differ significantly between the tin filter groups and the routine-dose groups (all $\mathrm{P}>0.05$ ). 
Table 2 Summary of the patient demographics and procedure performance in the tin filter and routine-dose groups

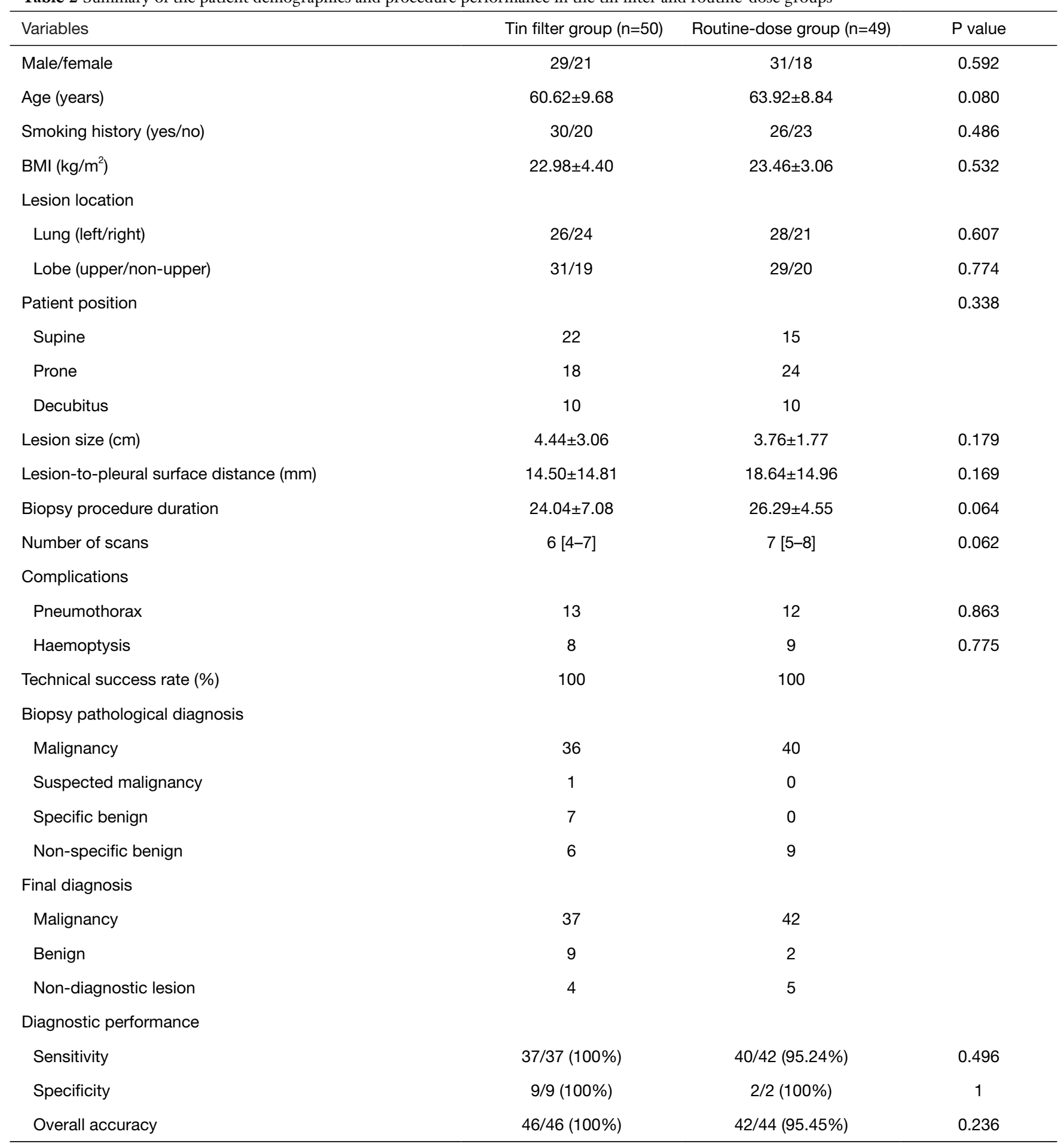

BMI, body mass index. 
Table 3 Subjective and objective image quality and metal artefact evaluation

\begin{tabular}{|c|c|c|c|c|c|c|c|c|c|}
\hline Parameters & \multicolumn{2}{|c|}{ Routine-dose protocol } & \multicolumn{2}{|c|}{ Tin filter protocol } & \multicolumn{5}{|c|}{$\mathrm{P}$} \\
\hline \multicolumn{10}{|c|}{ Subjective image quality } \\
\hline Rates & $3.49 \pm 0.65$ & $4.63 \pm 0.57$ & $3.88 \pm 0.66$ & $4.82 \pm 0.39$ & $<0.001$ & $<0.001$ & $<0.001$ & 0.03 & 0.188 \\
\hline CNR & $103.49 \pm 20.88$ & $102.99 \pm 19.43$ & $64.82 \pm 12.87$ & $64.58 \pm 12.37$ & $<0.001$ & 0.391 & 0.425 & $<0.001$ & $<0.001$ \\
\hline FOM & $14,041 \pm 7,230$ & $13,836 \pm 6,849$ & $21,866 \pm 10,656$ & $21,639 \pm 9,964$ & $<0.001$ & 0.263 & 0.416 & 0.001 & 0.001 \\
\hline CTNR (HU) & $246.51 \pm 155.59$ & $147.55 \pm 125.81$ & $158.10 \pm 94.32$ & $116.62 \pm 103.48$ & 0.001 & $<0.001$ & 0.001 & 0.017 & 0.558 \\
\hline
\end{tabular}

Group A: routine-dose without iMAR; Group B: routine-dose with iMAR; Group C: tin filter without iMAR; Group D: tin filter with iMAR; CNR, contrast-to-noise ratio; FOM, figure-of-merit; CTNR, CT number reduction; MDB, metal diameter blurring.

Table 4 Retest reliability interobserver agreement for subjective evaluation, CTNR and MDB

\begin{tabular}{lccc}
\hline Group & CTNR & MDB & Rates \\
\hline Group A & $0.898(0.83-0.94)$ & $0.910(0.85-0.95)$ & $0.893^{\mathrm{a}}$ \\
Group B & $0.852(0.75-0.91)$ & $0.867(0.78-0.92)$ & $0.874^{\mathrm{a}}$ \\
Group C & $0.853(0.76-0.91)$ & $0.917(0.86-0.95)$ & $0.927^{\mathrm{a}}$ \\
Group D & $0.880(0.80-0.93)$ & $0.912(0.85-0.95)$ & $0.935^{\mathrm{a}}$ \\
\hline
\end{tabular}

Group A: routine-dose without iMAR; Group B: routine-dose with iMAR; Group C: tin filter without iMAR; Group D: tin filter with iMAR. The retest reliability for CTNR and MDB was assessed using the interclass correlation coefficient (ICC). ${ }^{\text {a }}$, interobserver agreement for the qualitative image quality among the three readers was assessed using the weighted kappa (к). CTNR, CT number reduction; MDB, metal diameter blurring.

\section{Subjective image quality evaluation}

The qualitative image quality assessment scores given by both readers were significantly higher for the tin filter groups than for the routine-dose groups (group A vs. group $\mathrm{C}, \mathrm{P}=0.03$; group $\mathrm{B} v$ s. group $\mathrm{D}, \mathrm{P}=0.188$; Table 3). These scores showed excellent interobserver agreement $(\kappa=0.87$ 0.94 for each group; Table 4).

The scores for images with iMAR were higher than those without iMAR (group A vs. group B, or group C vs. group D, adjusted $\mathrm{P}<0.01$ ), which suggested that the qualitative image quality of CT with iMAR was superior to that without iMAR (Figures 2,3).

\section{Objective image quality evaluation}

The objective image quality results are summarised in
Table 3 and visualised in Figure 4. In the dose-related image analysis, the mean image noise in the tin filter groups was higher than that in the routine-dose groups (group A vs. group $\mathrm{C}$, and group $\mathrm{B} v s$. group $\mathrm{D}$, all $\mathrm{P}<0.05$ ). The mean CNR in the tin filter groups was lower than that in the routine-dose groups, while the mean FOM was higher in the tin filter groups than in the routine-dose groups (group A vs. group C, or group B vs. group $\mathrm{D}$; all $\mathrm{P}<0.05$ ).

\section{Metal artefact evaluation}

The metal diameter blurring (MDB) values in the tin filter groups with and without iMAR were 0.85 and 2.26, respectively $(\mathrm{P}<0.01$, Table 3 and Figure 4$)$, with a true needle width of $1.4 \mathrm{~mm}$; for the routine-dose groups, the metal diameter blurring (MDB) values were 1.25 and 3.97 with and without iMAR, respectively $(\mathrm{P}<0.01)$. The CT 

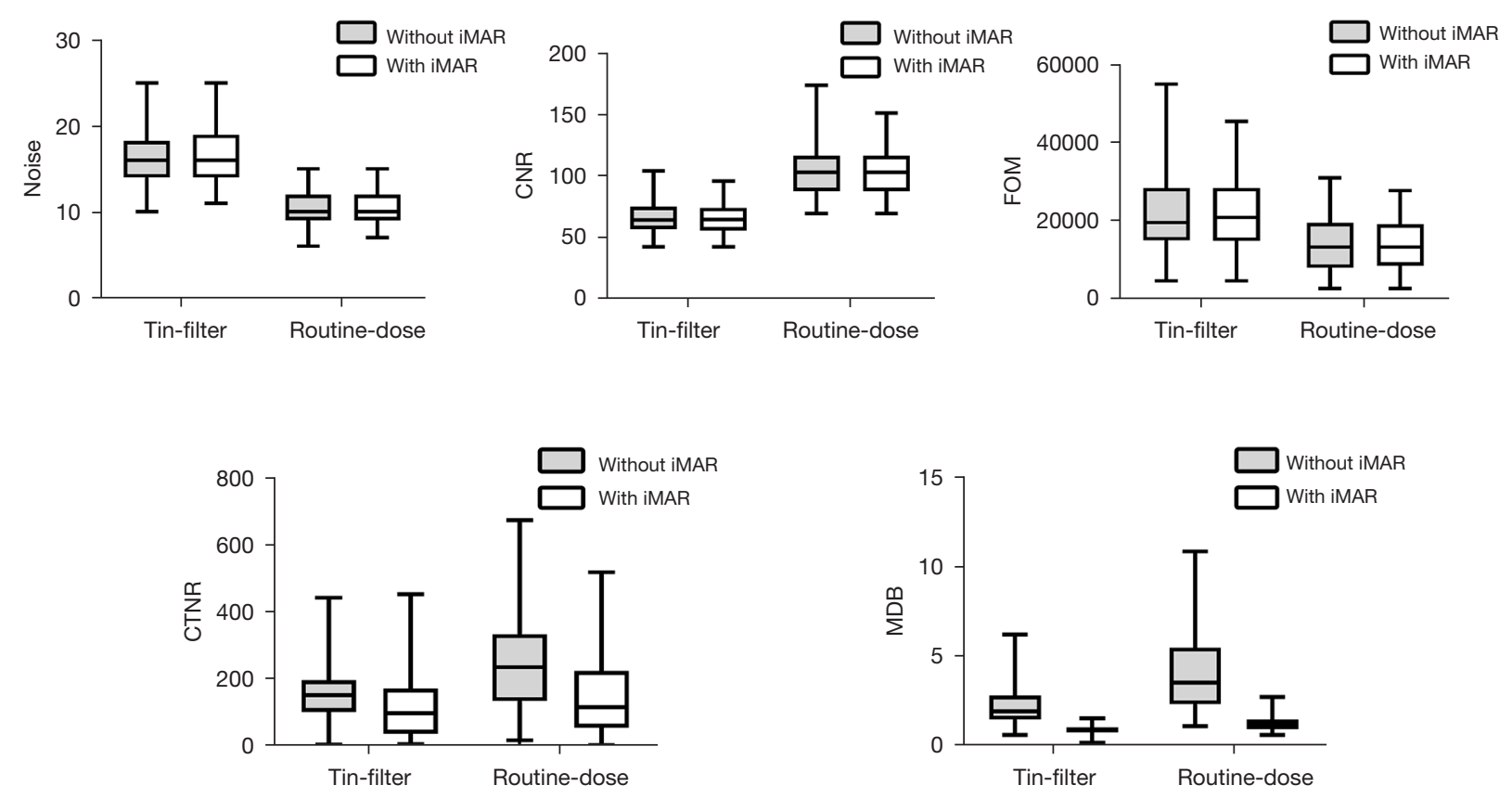

Figure 4 Comparison of quantitative image analyses (noise, CNR, FOM, CTNR, and MDB) among the four groups. The tin filter group with iMAR achieved a significant reduction in metal artefacts (lowest CTNR and MDB) and a higher dose efficiency (highest FOM). CNR, contrast-to-noise ratio; FOM, figure-of-merit; CTNR, CT number reduction; MDB, metal diameter blurring.

Table 5 Dose comparison between the tin filter and routine-dose groups per scan

\begin{tabular}{lccc}
\hline Parameters & Routine-dose protocol & Tin filter protocol & $P$ \\
\hline CTDlvol (mGy) & $5.56 \pm 1.86$ & $1.47 \pm 0.50$ & $<0.001$ \\
DLP $(\mathrm{mGy} \cdot \mathrm{cm})$ & $67.99 \pm 32.11$ & $16.32 \pm 7.75$ & $<0.001$ \\
Estimated ED $(\mathrm{mSv})$ & $0.95 \pm 0.45$ & $0.23 \pm 0.11$ & $<0.001$ \\
Effective mAs & $51.84 \pm 17.37$ & $50.44 \pm 16.26$ & 0.680 \\
\hline
\end{tabular}

CTDIvol, CT dose index; DLP, dose length product; ED, effective dose.

number reduction (CTNR) values in the tin filter group with and without iMAR were 116.62 and $158.10 \mathrm{HU}$, respectively $(\mathrm{P}<0.01)$; for the routine-dose groups, the CTNR values were 147.55 and $246.51 \mathrm{HU}$ with and without iMAR, respectively $(\mathrm{P}<0.01)$. In images without iMAR reconstruction, the tin filter group was slightly superior to the routine-dose group $(\mathrm{P}=0.017)$. The ICC for the CTNR and MDB in all groups showed excellent agreement (Table 4).

\section{Radiation dose}

Compared with the routine-dose groups, the tin filter groups had a significantly lower CTDIvol $(1.47 \pm 0.50 \mathrm{vs}$. 5.56 $\pm 1.86 ; \mathrm{P}<0.001)$, DLP $(16.32 \pm 7.75$ vs. $67.99 \pm 32.11$; $\mathrm{P}<0.001)$, and $\mathrm{ED}(0.23 \pm 0.11$ vs. $0.95 \pm 0.45 ; \mathrm{P}<0.001$; Table $5)$. However, no statistically significant difference was found in the effective milliampere-seconds ( $\mathrm{mAs}$ ) between the groups $(\mathrm{P}=0.680)$.

The radiation ED in the tin filter groups was $73 \%$ lower than that in the routine-dose groups, and all the doserelated parameters were obtained for each scan.

\section{Diagnostic performance and complication rates}

A $100 \%$ technical success rate was achieved in all groups 
(Table 2), with each patient undergoing a biopsy for a single target lesion. The tin filter and routine-dose biopsy procedures had an average duration of $24.04 \pm 7.08$ and $26.29 \pm 4.55$ min, respectively $(\mathrm{P}=0.064)$.

Pneumothorax developed in $26.0 \%$ and $24.5 \%$ of patients in the tin filter and routine-dose groups, respectively $(\mathrm{P}=0.863$; Table 2$)$. Haemoptysis developed in $16.0 \%$ and $18.4 \%$ of patients in the tin filter and routinedose groups, respectively ( $\mathrm{P}=0.775$; Table 2$)$.

Patients with non-diagnostic lesions were not included in the calculation of diagnostic performance. In the tin filter groups, the sensitivity, specificity, and accuracy for the diagnosis of malignancy were all 100\% (37/37, 9/9, and $46 / 46$, respectively); in the routine-dose groups, these values were $95.24 \%(40 / 42), 100 \%(2 / 2)$, and $95.45 \%$ (42/44), respectively. The overall diagnostic sensitivity, specificity, and accuracy for pulmonary lesions did not differ significantly between the groups $(\mathrm{P}=0.496,1.0$, and 0.236 , respectively; Table 2).

\section{Discussion}

Lung biopsies are primarily performed to differentiate between benign and malignant lung lesions. In patients with malignant lung lesions, they allow for a more definitive pathological diagnosis and assessment of specific molecular markers (18). In this study, we discussed a method that uses tin filtration combined with an iMAR algorithm to reduce the radiation dose and metal artefacts during CT-guided lung biopsy. Both subjective and objective assessments of image quality demonstrated that a tin filter combined with iMAR improved diagnostic confidence and provided better radiation dose efficiency than the routine-dose CT protocol.

During a CT-guided biopsy, iMAR reconstruction can improve the display of lesions when metal artefacts significantly limit their visibility (15). Our results indicated that images obtained with the iMAR algorithm showed a significant decrease in the degree of artefacts compared to images without iMAR. The overall image quality was significantly improved with the iMAR algorithm. Detailed analysis revealed that using iMAR with tin filtration led to slight artefact reduction in comparison to using a tin filter protocol without $\mathrm{MMAR}$, and that using iMAR with a routine-dose protocol resulted in strong artefact reduction in comparison to a routine-dose protocol without iMAR. Previous studies $(13,15,19-21)$ have reported a reduction in metal artefacts from metal needles in CT-guided microwave ablation, metallic thoracic implants, hip prostheses, dental hardware, and spinal implants when iMAR is used, which is consistent with our results for metal needles in lung biopsy.

Tin filtration has the potential to reduce the radiation dose. Previous studies $(22,23)$ used a tin filter to reduce the radiation dose during CT-guided biopsy procedures using $100 \mathrm{kVp}$ in a third-generation dual-source CT system, with a dose reduction of $90 \%$. Compared with the routinedose protocol, our low-dose protocol with a tin filter at $130 \mathrm{kVp}$ yielded a $73.2 \%$ reduction per scan, which is consistent with the radiation dose reductions of 53.3-94.5\% reported in previous studies. Lowering the tube voltage and decreasing the tube current are effective methods for reducing the radiation dose $(4,6,16,24-26)$. The mean DLP and estimated ED are significantly reduced using the low tube voltage protocol and low tube current. Fu et al. reported that a $120-\mathrm{kVp}$ protocol $(15 \mathrm{mAs})$ yielded a $90 \%$ reduction in the radiation dose ( $0.5 v s .5 .1 \mathrm{mSv})$ compared to a $120-\mathrm{kVp}$ protocol $(150 \mathrm{mAs})(4)$, which is slightly larger than the reduction in this study. A possible explanation for this difference is that we used a kilovoltage of $130 \mathrm{kV}$ with the tin filter, and a higher kilovoltage may deliver higher radiation doses than a lower kilovoltage.

In this study, using tin filtration combined with an iMAR algorithm in CT-guided lung biopsies was technically successful in $100 \%$ of cases. This observation is consistent with the rates reported in previous studies (98.2-100\%) $(4,22)$. No significant difference was observed in the rates of procedure-associated pneumothorax or haemoptysis between the groups, which suggests that the low-dose protocol using tin filtration combined with an iMAR algorithm does not increase the risk of either of these biopsy-associated complications, a finding that is consistent with those of previous studies $(27,28)$. Although the CNR of the tin filter groups was significantly lower than that of the routine-dose groups, the technical success rate was identical to that of the routine-dose groups. The reason for this may be that, unlike in routine diagnostic imaging, detailed lesion features are not required during the biopsy procedure (22). Additionally, in terms of diagnostic accuracy, no statistically significant differences were found in the sensitivity or overall diagnostic accuracy between the groups, a finding that was consistent with those of previous studies $(4,6,22)$. Overall, our results demonstrated no obvious reduction in the diagnostic accuracy of low-dose CT using a tin filter combined with an iMAR algorithm, suggesting that the overall image quality was sufficient for 
the biopsy procedures. However, as phantom and patient studies $(29,30)$ have shown that a sensitivity of almost $100 \%$ for sub-solid nodules can be reached using a low-dose tin filter protocol, we did not discuss the issues in detecting ground-glass nodules.

This study has several limitations. First, some previous studies $(4,22,23)$ reported a slightly higher radiation dose reduction than ours using the tin filter technique. The cause for this difference might be that those studies used a third-generation dual-source system, which contains more advanced hardware and software than our normal clinicaluse CT scanner. Furthermore, the kilovolt setting was higher in our study due to the image quality requirements of the intervention operators at our institution. Future studies should consider using a lower kilovolt setting to further reduce the radiation dose, depending on patients' BMIs (11). Second, due to workplace arrangements, several intervention operators were involved in our study, which is a potential cause of bias in our findings. Third, we did not use CT fluoroscopy in our study. CT fluoroscopy has become popular recently, because it has been shown to significantly reduce the total procedure time and radiation dose for patients while providing higher diagnostic accuracy $(31,32)$. However, a fundamental problem with CT fluoroscopy is that the physicians need to stay in the examination room during the scanning process and are exposed to radiation (33). Fourth, due to ethical requirements, we did not perform a comparative study of the success rate of biopsy and the incidence of complications using iMAR, because this technique can be applied during the biopsy process in real-time without additional operations. Fifth, owing to the limitation of the scanning range and individual heterogeneity, lung tissue could not be obtained from the same area in different patients, and background noise was defined as the air noise outside the thorax rather than the air noise inside the tracheal lumen. Sixth, the trial was limited by its sample size. Further prospective randomised controlled trials with larger sample sizes are needed in future to better understand the viability, safety, and diagnostic accuracy of using low-dose CT guidance in the performance of lung biopsies.

In conclusion, tin filtration combined with an iMAR algorithm may largely reduce the radiation dose compared to a routine-dose $\mathrm{CT}$ protocol, while maintaining a comparable image quality and incidence of complications and producing fewer metal artefacts. This low-dose protocol could replace the conventional protocol as the routine protocol for CT-guided lung biopsy.

\section{Acknowledgments}

The authors thank all volunteers who participated in the study and the staff of the Department of Radiology, Chongqing University Cancer Hospital \& Chongqing Cancer Institute \& Chongqing Cancer Hospital in Chongqing, China for their selfless and valuable assistance. Funding: This study received funding from the National Natural Science Foundation of China (Grant No. 82071883 ), the Combination Projects of Medicine and Engineering of the Fundamental Research Funds for the Central Universities in 2019 (Project No. 2019CDYGYB008), the Chongqing Key Medical Research Project of the Combination of Science and Medicine (Grant No. 2019ZDXM007), and the 2019 SKY Imaging Research Fund of the Chinese International Medical Foundation (Project No. Z-2014-07-1912-10).

\section{Footnote}

Reporting Checklist: The authors have completed the STARD reporting checklist. Available at https://dx.doi. org/10.21037/qims-21-555

Conflicts of Interest: All authors have completed the ICMJE uniform disclosure form (available at https://dx.doi. org/10.21037/qims-21-555). The authors have no conflicts of interest to declare.

Ethical Statement: The authors are accountable for all aspects of the work in ensuring that questions related to the accuracy or integrity of any part of the work are appropriately investigated and resolved. The study was conducted in accordance with the Declaration of Helsinki (as revised in 2013). This study was approved by the ethics committee of Chongqing university cancer hospital (No. CZLS2021182-A), and all the patients provided written informed consent after receiving a detailed explanation of the procedure.

Open Access Statement: This is an Open Access article distributed in accordance with the Creative Commons Attribution-NonCommercial-NoDerivs 4.0 International License (CC BY-NC-ND 4.0), which permits the noncommercial replication and distribution of the article with the strict proviso that no changes or edits are made and the original work is properly cited (including links to both the formal publication through the relevant DOI and the license). See: https://creativecommons.org/licenses/by-nc-nd/4.0/. 


\section{References}

1. Bray F, Ferlay J, Soerjomataram I, Siegel RL, Torre LA, Jemal A. Global cancer statistics 2018: GLOBOCAN estimates of incidence and mortality worldwide for 36 cancers in 185 countries. CA Cancer J Clin 2018;68:394-424.

2. Wu CC, Maher MM, Shepard JA. CT-guided percutaneous needle biopsy of the chest: preprocedural evaluation and technique. AJR Am J Roentgenol 2011;196:W511-4.

3. Mountford PJ, Temperton DH. Recommendations of the International Commission on Radiological Protection (ICRP) 1990. Eur J Nucl Med 1992;19:77-9.

4. Fu YF, Li GC, Xu QS, Shi YB, Wang C, Wang T. Computed tomography-guided lung biopsy: a randomized controlled trial of low-dose versus standard-dose protocol. Eur Radiol 2020;30:1584-92.

5. Cordts I, Deschauer M, Lingor P, Burian E, Baum T, Zimmer C, Maegerlein C, Sollmann N. Radiation dose reduction for $\mathrm{CT}$-guided intrathecal nusinersen administration in adult patients with spinal muscular atrophy. Sci Rep 2020;10:3406.

6. Lee HN, Lee SM, Choe J, Lee SM, Chae EJ, Do KH, Seo JB. Diagnostic performance of CT-guided percutaneous transthoracic core needle biopsy using low tube voltage $(100 \mathrm{kVp})$ : comparison with conventional tube voltage (120kVp). Acta Radiol 2018;59:425-33.

7. Baumueller S, Winklehner A, Karlo C, Goetti R, Flohr T, Russi EW, Frauenfelder T, Alkadhi H. Low-dose CT of the lung: potential value of iterative reconstructions. Eur Radiol 2012;22:2597-606.

8. Chang DH, Hiss S, Mueller D, Hellmich M, Borggrefe J, Bunck AC, Maintz D, Hackenbroch M. Radiation Dose Reduction in Computed Tomography-Guided Lung Interventions using an Iterative Reconstruction Technique. Rofo 2015;187:906-14.

9. Dewes P, Frellesen C, Scholtz JE, Fischer S, Vogl TJ, Bauer RW, Schulz B. Low-dose abdominal computed tomography for detection of urinary stone disease Impact of additional spectral shaping of the $\mathrm{X}$-ray beam on image quality and dose parameters. Eur J Radiol 2016;85:1058-62.

10. Vivier S, Deken V, Arous Y, Faivre JB, Duhamel A, Deschildre A, Flohr T, Remy J, Remy-Jardin M. Pediatric chest computed tomography at $100 \mathrm{kVp}$ with tin filtration: comparison of image quality with $70-\mathrm{kVp}$ imaging at comparable radiation dose. Pediatr Radiol 2020;50:188-98.

11. Li J, Mai Z, Zhang Z, Cui J, Yang M, Ma X, Wang Y. Chest $\mathrm{CT}$ screening in patients with overweight or obesity using spectral shaping at $150 \mathrm{kVp}$ : compared with $120 \mathrm{kVp}$ protocol and spectral shaping at $100 \mathrm{kVp}$ protocol. Jpn J Radiol 2020;38:451-7.

12. Zhou W, Bartlett DJ, Diehn FE, Glazebrook KN, Kotsenas AL, Carter RE, Fletcher JG, McCollough CH, Leng S. Reduction of Metal Artifacts and Improvement in Dose Efficiency Using Photon-Counting Detector Computed Tomography and Tin Filtration. Invest Radiol 2019;54:204-11.

13. Trabzonlu TA, Terrazas M, Mozaffary A, Velichko YS, Yaghmai V. Application of Iterative Metal Artifact Reduction Algorithm to CT Urography for Patients With Hip Prostheses. AJR Am J Roentgenol 2020;214:137-43.

14. Aissa J, Thomas C, Sawicki LM, Caspers J, Kröpil P, Antoch G, Boos J. Iterative metal artefact reduction in CT: can dedicated algorithms improve image quality after spinal instrumentation? Clin Radiol 2017;72:428.e7-428.e12.

15. Do TD, Melzig C, Vollherbst DF, Pereira PL, Kauczor HU, Kachelrieß M, Sommer CM. The value of iterative metal artifact reduction algorithms during antenna positioning for CT-guided microwave ablation. Int $\mathrm{J}$ Hyperthermia 2019;36:1223-32.

16. Wagner MG, Hinshaw JL, Li Y, Szczykutowicz TP, Laeseke P, Mistretta CA, Lee FT Jr. Ultra-Low Radiation Dose CT Fluoroscopy for Percutaneous Interventions: A Porcine Feasibility Study. Radiology 2019;291:241-9.

17. Huang JY, Kerns JR, Nute JL, Liu X, Balter PA, Stingo FC, Followill DS, Mirkovic D, Howell RM, Kry SF. An evaluation of three commercially available metal artifact reduction methods for CT imaging. Phys Med Biol 2015;60:1047-67.

18. Prosch H, Stadler A, Schilling M, Bürklin S, Eisenhuber E, Schober E, Mostbeck G. CT fluoroscopy-guided vs. multislice CT biopsy mode-guided lung biopsies: accuracy, complications and radiation dose. Eur J Radiol 2012;81:1029-33.

19. Aissa J, Boos J, Schleich C, Sedlmair M, Krzymyk K, Kröpil P, Antoch G, Thomas C. Metal Artifact Reduction in Computed Tomography After Deep Brain Stimulation Electrode Placement Using Iterative Reconstructions. Invest Radiol 2017;52:18-22.

20. Diehn FE, Michalak GJ, DeLone DR, Kotsenas AL, Lindell EP, Campeau NG, Halaweish AF, McCollough CH, Fletcher JG. CT Dental Artifact: Comparison of an Iterative Metal Artifact Reduction Technique with Weighted Filtered Back-Projection. Acta Radiol Open 2017;6:2058460117743279.

21. Aissa J, Boos J, Sawicki LM, Heinzler N, Krzymyk K, 
Sedlmair M, Kröpil P, Antoch G, Thomas C. Iterative metal artefact reduction (MAR) in postsurgical chest CT: comparison of three iMAR-algorithms. Br J Radiol 2017;90:20160778.

22. Li C, Liu B, Meng H, Lv W, Jia H. Efficacy and Radiation Exposure of Ultra-Low-Dose Chest CT at $100 \mathrm{kVp}$ with Tin Filtration in CT-Guided Percutaneous Core Needle Biopsy for Small Pulmonary Lesions Using a ThirdGeneration Dual-Source CT Scanner. J Vasc Interv Radiol 2019;30:95-102.

23. Liu B, Fang J, Jia H, Sun Z, Liao J, Meng H, Pan F, Li C. Ultralow dose computed tomography protocol for hookwire localization of solitary pulmonary nodules prior to video-assisted thoracoscopic surgery. Thorac Cancer 2019;10:1348-54.

24. Alagic Z, Alagic H, Bujila R, Srivastava S, Jasim S, Lindqvist $M$, Wick MC. First experiences of a lowdose protocol for CT-guided musculoskeletal biopsies combining different radiation dose reduction techniques. Acta Radiol 2020;61:28-36.

25. Guberina N, Forsting M, Ringelstein A, Suntharalingam S, Nassenstein K, Theysohn J, Wetter A. Radiation exposure during CT-guided biopsies: recent CT machines provide markedly lower doses. Eur Radiol 2018;28:3929-35.

26. Greffier J, Hoballah A, Sadate A, de Oliveira F, Claret PG, de Forges H, Loubet P, Mauboussin JM, Hamard A, Beregi JP, Frandon J. Ultra-low-dose chest CT performance for the detection of viral pneumonia patterns during the COVID-19 outbreak period: a monocentric experience. Quant Imaging Med Surg 2021;11:3190-9.

27. Zhu J, Qu Y, Wang X, Jiang C, Mo J, Xi J, Wen Z. Risk factors associated with pulmonary hemorrhage and hemoptysis following percutaneous CT-guided transthoracic lung core needle biopsy: a retrospective study of 1,090 cases. Quant Imaging Med Surg 2020;10:1008-20.

28. Aktaş AR, Gözlek E, Y1lmaz Ö, Kayan M, Ünlü N, Demirtaş H, Değirmenci B, Kara M. CT-guided transthoracic biopsy: histopathologic results and complication rates. Diagn Interv Radiol 2015;21:67-70.

29. Martini K, Ottilinger T, Serrallach B, Markart S, Glaser-Gallion N, Blüthgen C, Leschka S, Bauer RW, Wildermuth S, Messerli M. Lung cancer screening with submillisievert chest CT: Potential pitfalls of pulmonary findings in different readers with various experience levels. Eur J Radiol 2019;121:108720.

30. Messerli M, Kluckert T, Knitel M, Wälti S, Desbiolles L, Rengier F, Warschkow R, Bauer RW, Alkadhi H, Leschka $\mathrm{S}$, Wildermuth S. Ultralow dose CT for pulmonary nodule detection with chest $\mathrm{x}$-ray equivalent dose - a prospective intra-individual comparative study. Eur Radiol 2017;27:3290-9.

31. Carlson SK, Bender CE, Classic KL, Zink FE, Quam JP, Ward EM, Oberg AL. Benefits and safety of CT fluoroscopy in interventional radiologic procedures. Radiology 2001;219:515-20.

32. Yoon SH, Lee SM, Park CH, Lee JH, Kim H, Chae KJ, Jin KN, Lee KH, Kim JI, Hong JH, Hwang EJ, Kim H, Suh YJ, Park S, Park YS, Kim DW, Choi M, Park CM. 2020 Clinical Practice Guideline for Percutaneous Transthoracic Needle Biopsy of Pulmonary Lesions: A Consensus Statement and Recommendations of the Korean Society of Thoracic Radiology. Korean J Radiol 2021;22:263-80.

33. Paulson EK, Sheafor DH, Enterline DS, McAdams HP, Yoshizumi TT. CT fluoroscopy--guided interventional procedures: techniques and radiation dose to radiologists. Radiology 2001;220:161-7.
Cite this article as: Zhang $\mathrm{J}$, Liu M, Liu D, Li X, Lin M, Tan Y, Luo Y, Zeng X, Yu H, Shen H, Wang X, Liu L, Tan Y, Zhang J. Low-dose CT with tin filter combined with iterative metal artefact reduction for guiding lung biopsy. Quant Imaging Med Surg 2022;12(2):1359-1371. doi: 10.21037/qims-21-555 